\title{
A Context-Aware Method for Authentically Simulating Outdoors Shadows for Mobile Augmented Reality
}

\author{
João Barreira, Maximino Bessa, Luís Barbosa, and Luís Magalhães
}

\begin{abstract}
Visual coherence between virtual and real objects is a major issue in creating convincing augmented reality (AR) applications. To achieve this seamless integration, actual light conditions must be determined in real time to ensure that virtual objects are correctly illuminated and cast consistent shadows. In this paper, we propose a novel method to estimate daylight illumination and use this information in outdoor AR applications to render virtual objects with coherent shadows. The illumination parameters are acquired in real time from context-aware live sensor data. The method works under unprepared natural conditions. We also present a novel and rapid implementation of a state-of-the-art skylight model, from which the illumination parameters are derived. The Sun's position is calculated based on the user location and time of day, with the relative rotational differences estimated from a gyroscope, compass and accelerometer. The results illustrated that our method can generate visually credible AR scenes with consistent shadows rendered from recovered illumination.
\end{abstract}

Index Terms-Augmented reality, context-awareness, shadows coherence, photometric registration

\section{INTRODUCTION}

$\mathrm{T}_{\mathrm{t}}^{\mathrm{H}}$ He objective of augmented reality (AR) technology is to interactively display virtual objects in a real environment and in real time [1]. Many AR applications in such fields as cultural heritage, architectural visualization and building engineering require that the virtual objects appear as real as possible to the user. Ideally, the user should not be able to distinguish between virtual and real. This problem is mainly addressed by geometric registration and credible illumination. The geometric registration issue has been widely researched in the literature, and recent results have shown that current methods are sufficiently accurate to align virtual objects in the real environment without preparation [2]. The major issue that must be addressed to build realistic AR applications is rendering virtual objects with correct illumination. This task implies estimating the real-world illumination and handling any changes in the scene, which is known as photometric registration.

In recent years, several solutions have been proposed to address this problem. However, the prominent approaches either require a 3D model of the scene geometry [3] or are expensive and require special equipment, such as a light probe [4] or fisheye camera lens [5]. Although the use of light probes provides precise information regarding illumination conditions in terms of radiance and the incoming direction, these methods have some serious drawbacks. First, a light probe image requires multiple exposures of the same scene because it must be in high dynamic range (HDR) format. Furthermore, the light probe information

- J. Barreira, M. Bessa, and L. Barbosa are with the Universidade de Trásos-Montes e Alto Douro, Vila Real, Portugal, and INESC TEC, Porto, Portugal. E-mail: jcsbarreira@gmail.com, \{maxbessa, lfb\}@utad.pt

- L. Magalhães is with the Centro Algoritmi / Universidade do Minho, Braga, Portugal. E-mail:Imagalhaes@dsi.uminho.pt in dynamic scenes becomes invalid when changes occur in the illumination conditions unless constantly observing the light probe with a camera and tracking algorithm (as in Aittala [6]), which is computationally demanding. This method is also not suitable for unprepared environments because the coordinate system of the light probe must be calibrated. Another approaches for estimating the lighting from images of a scene using inverse rendering requires complete reconstruction of the scene geometry.

This paper presents a new photometric registration method for estimating sunlight direction and intensity based on information from sensors. The method is applied to outdoor AR to render consistent shadows. In our system, we use an ambient light sensor embedded in current mobile phones to automatically detect the absolute scene illuminance, from which the sky and Sun parameters are derived depending on the current weather conditions. The position of the Sun relative to the user's location and orientation is computed as a directional light based on the geospatial position of the device and the current date and time. The calculated scene illuminance is then used to render the virtual objects, which results in a visually convincing scene.

The proposed method is implemented on mobile devices, such as smartphones and tablets, because they are equipped with GPUs, support a variety of wireless communication (GSM, Wi- Fi) and include a growing set of integrated sensors, such as an accelerometer, gyroscope, global positioning system (GPS) and ambient light sensor (ALS). The main contribution of our work includes a novel system of illumination estimation capable of considering changes in the light-scene conditions without any additional intrusive objects. The system presents a new concept to estimate diffuse and direct transmittance of skylight that is easily implemented on mobile devices because it only requires live data from embedded sensors. 


\section{BACKGROUND AND RELATED WORK}

Photometric registration refers to the procedure by which real-world lighting is estimated. The main approaches proposed in the literature assume that either a 3D geometric model of the scene or an environment map obtained from a light probe or HDR camera is available. Aittala [6] used a diffuse light probe to estimate real-world lighting conditions based on $l_{1}$-regularized least-squares minimization. Their method allows light distribution to be accurately determined but is computationally demanding owing to the dense directional sampling required. Calian et al. [7] proposed a method to estimate the illumination based on the shading parameterized by the surface orientation and visibility. In their method, the user must rotate the camera manually around the probe. Another method presented by Knecht et al. [8] derives the dynamic real illumination from the images captured using a fish-eye lens camera, assuming that a geometric model of the real scene is available and that the basic material properties of real objects are also known.

Alternatively, Kan and Kaufmann [9] presented a method restricted to simple scenes that computes arbitrary and refractive effects using fully recursive raytracing. Karsch et al. [10], [11] proposed a system to simulate global illumination that supports drag-and-drop 3D object insertion into existing photographs. The probeless system of Jachnik et al. [12] captures the surface light fields to create an environmental map. However, their work was limited to static environments because the user is required to scan a target object and cannot consider illumination changes in real time.

Few studies have focused on outdoor photometric registration. Lalonde et al. [13] estimated the illumination conditions of a single outdoor image from the sky, ground, and vertical surfaces in the image. Their method only estimates the position of the Sun and cannot be used in AR to insert virtual objects into real camera images. Madsen et al. [14] proposed a method to estimate the Sun and sky illumination from shadows on the ground that requires a stereo camera to recover the depth information. Furthermore, the real-world scene must contain dynamic objects. Wang and Samaras [15] also presented a method for estimating multiple light sources from shadows. However, their approach has only been used in static images. Sato et al. [16] recovered the scene lighting from image brightness inside shadows cast by an object of known geometry and location.

Other approaches, such as that presented by Liu et al. [17] have proposed estimating outdoor lighting conditions by exploiting the relation between image statistics and lighting parameters. The approach of Liu et al. is limited to a fixed viewpoint. Recently, Liu and Granier [18] proposed a computationally demanding method to estimate illumination intensities based on a set of planar feature points extracted from the scene video. Knorr and Kurz [19] presented an algorithm for the coherent rendering of virtual augmentations based on illumination estimation from the intensities of sample points in a frontal face. However, their method can only be used on front-facing surfaces.

Methods partially based on Kinect Fusion allow for the full geometric reconstruction of the scene but are excessively detailed and thus excessively noisy in the depth map if the intention is to recover irradiance information. Finally, Gruber et al. [20], [21] presented a method of illumination estimation by projecting a low-frequency function into $\mathrm{SH}$ coefficients for indoor scenarios.

\section{CONTEXT-AWARENESS FOR ILLUMINATION ESTI- MATION}

This section describes the proposed approach to estimate the scene illuminance owing to the Sun and sky. The main process is to recover the daylight illumination contributions, which depend on current weather conditions. Instead of using computer vision methods to derive information on the scene illumination from the camera image, we propose to use the current date and time as well as GPS to determine where the Sun is located relative to the device. We also use a weather API to determine whether the sky is clear, partly cloudy, or cloudy, and we employ the ALS of the mobile device to measure the absolute illuminance.

Figure 1 provides an overview of the scene illuminance estimation pipeline, which consists of three main steps, namely, reconstruction of the Sun's position and direction, estimation of the illuminance parameters and detection of dynamic shadows. This pipeline is applied at each frame. An ALS change sensitivity is used so that only when there is a significant change on its value the pipeline is executed. In the current implementation, the ALS change sensitivity is specified as 5.0. The value is interpreted as a percentage, and the data-updated event is only executed when the lux value increases or decreases by $5 \%$. This reconstruction occurs every frame in real time and allows for dynamic changes in the actual light scene conditions. The following sections describe in more detail each step.

\subsection{Reconstruction of the Sun's position and direction}

We compute the Sun's position as a virtual directional light from the latitude, longitude, time and date using an implementation of the PSA algorithm [22]. The input is the location's latitude and longitude with the time converted to fractions of a degree. The azimuth angle is measured from the north and the zenith angle is measured from the vertical axis. The elevation angle is measured from the horizontal.

The equations from the Astronomical Almanac [23] can be used to calculate the ecliptic coordinates of the Sun.

$$
\begin{gathered}
\Omega=2.1429-0.0010394594 n \\
L=4.8950630+0.017202791698 n \\
g=6.2400600+0.0172019699 n
\end{gathered}
$$

$\Omega$ is the longitude of the perihelion, $L$ is the mean longitude of the Sun and $g$ is the mean anomaly. $n$ is the number of days since Greenwich noon. I.e the difference between the current Julian day, $J$, and Julian day 2451545.0 (noon 1 January 2000).

$$
n=J-2451545
$$




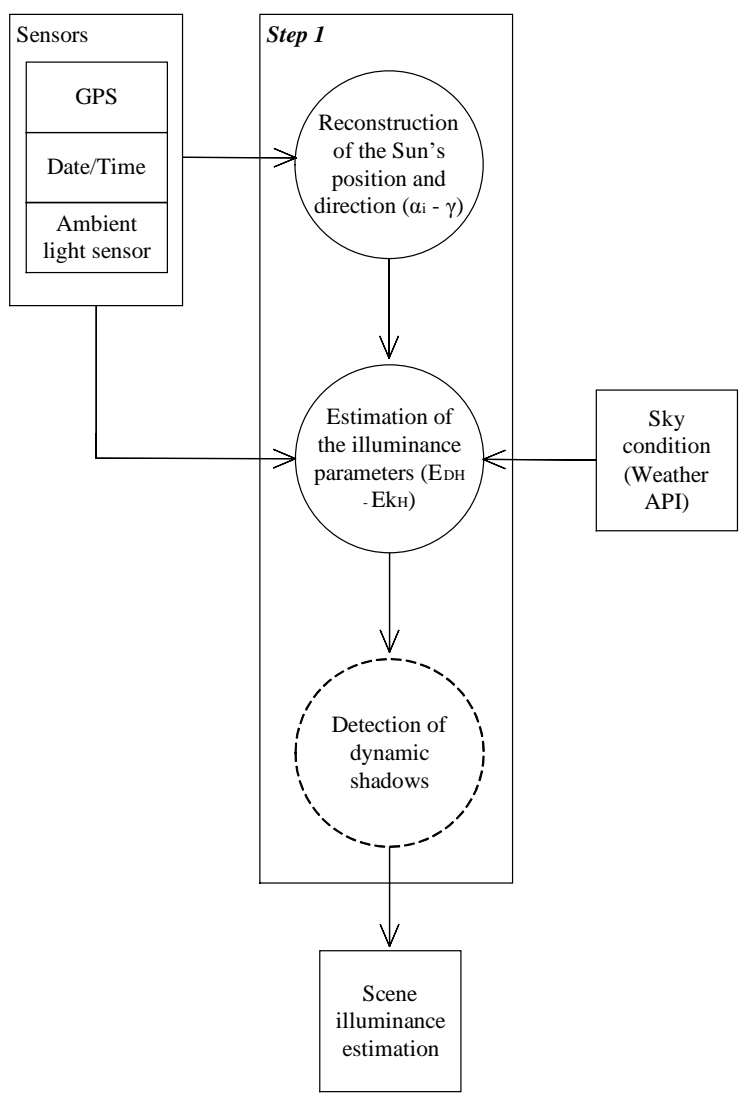

Fig. 1: Scene illuminance estimation pipeline. The current date and time and GPS location determine the Sun's position and direction. A weather API is used to determine the current sky conditions, and the ALS of the mobile device is used in the daylight illuminance calculation.

$J$ is computed from the current date and time using the following equation:

$$
\begin{array}{r}
J=(1461 x(y+4800+(m-14) / 12)) / 4 \\
+(367 x(m-2-12 x((m-14) / 12))) / 12 \\
-(3 x((y+4900+(m-14) / 12) / 100)) / 4 \\
+d-32075-0.5+\text { hour } / 24.0
\end{array}
$$

where $d, m$ and $y$ are current day, month and year respectively, and hour the hour of the day in Universal Time.

The ecliptic longitude $l$ and obliquity of the ecliptic $e p$ are given by

$$
\begin{gathered}
l=L+0.3341607 \sin (g)+0.00034894 \sin (2 g) \\
-0.0001134-0.0000203 \sin (\Omega) \\
e p=0.4090928-6.2140 \exp (-9) n+0.0000396 \cos (\Omega)
\end{gathered}
$$

Once the ecliptic longitude have been calculated, the Sun's right ascension $r a$ and declination $\delta$ can be obtained.

$$
\begin{aligned}
\delta & =\arcsin [\sin (e p) \sin (l)] \\
r a & =\arctan \left[\frac{\cos (e p) \sin (l)}{\cos (l)}\right]
\end{aligned}
$$

The conversion from celestial to horizontal coordinates are computed with the following equations

$$
\text { gmst }=6.697424324210+0657098283 n+\text { hour }
$$

$$
\begin{gathered}
\text { lmst }=(15 g m s t+\text { Long }) \times\left(\frac{\Pi}{180}\right) \\
w=\text { lmst }-r a
\end{gathered}
$$

where gmst is the Greenwich mean sidereal time, lmst is local mean sidereal time, and $w$ the hour angle.

The zenith distance, i.e. $90^{\circ}$ - altitude, is given by equation (13), where $\Phi$ is the latitude of the site.

$$
\theta=\arccos [\cos (\Phi) \cos (w) \cos (\delta)+\sin (\delta) \sin (\Phi)]
$$

The solar azimuth $\gamma$, which refers to the angle of the object around the horizon, is

$$
\gamma=\arctan \left[\frac{-\sin (w)}{\tan (\delta) \cos (\Phi)-\cos (w) \sin (\Phi)}\right]
$$

\subsection{Estimation of the illuminance parameters}

Because the direct solar illuminance is scattered by dust and other particles through the atmosphere, the entire sky dome also emits light. This scattered light depends on particular sky conditions: clear, partly cloudy and cloudy. Therefore, the absolute daylight illuminance includes direct horizontal illuminance $\left(E_{D H}\right)$ and diffused horizontal illuminance $\left(E_{K H}\right)$, and can be expressed as

$$
E=E_{D H}+E_{K H}
$$

In our approach, the absolute illuminance $(E)$ is obtained from the ALS measurement. The light sensor can detect this information in lux units.

The sky conditions are obtained using the user's specific location along with the date and time as input parameters to an online weather API-http://www.wunderground.com/. The system sends a URL request and receives the current weather condition (clear sky/partly cloudy sky/cloudy sky).

This information enables the estimation of the direct and diffuse illuminance contributions. For a particular sky condition, we implemented a sky ratio method based on IESNA recommended practice [24]. This is the ratio of the diffuse horizontal illuminance $E_{K H}$ to the absolute illuminance $E$.

$$
\left.\begin{array}{l}
\text { Clear sky: } E_{D H}=0.85 E \Rightarrow E_{K H}=0.15 E \\
\text { Partly cloudy sky: } E_{D H}=E_{K H}=0.5 E \\
\text { Cloudy sky: } E_{D H}=0 \Rightarrow E_{K H}=E
\end{array}\right\}
$$

Under clear sky conditions $E_{K H}<30 \% E$, and the Bird model [25] can provide a good estimation of the sky components on a horizontal surface. Accordingly, the direct illumination under this condition is $85 \%$ of the global scene illuminance. Under partly cloudy conditions, the direct and diffuse illuminance components range from $30 \%$ to $80 \%$ of the total scene illuminance. Based on practical purposes, it can be set to the average value of $50 \%$. Under cloudy sky conditions, there is no direct horizontal illuminance from the Sun, and thus, only the horizontal sky illuminance is distributed around the hemisphere. 


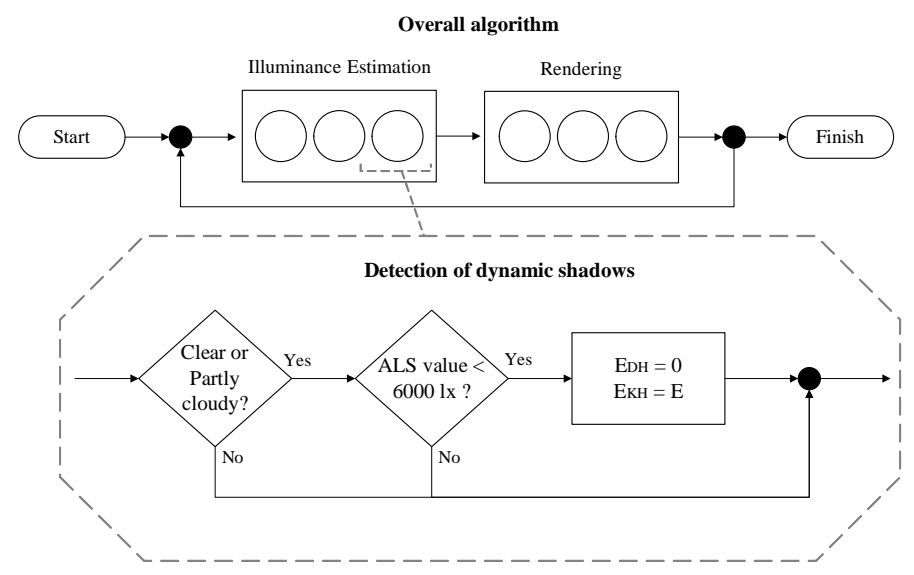

Fig. 2: Detection of dynamic shadows algorithm fluxogram.

\subsection{Detection of dynamic shadows}

We use the absolute illuminance values reading from the ALS to detect if the object is under direct sunlight or in the shadow. This detection is only performed if the sky condition is clear or partly cloudy since, if the sky condition is cloudy, by equation (16) $E_{D H}=0$, so no shadow is generated. At every frame the absolute illuminance value reading from the ALS is verified. A virtual object is considered as in shadow if the absolute illuminance is less than 6,000 lx, which is the minimum available outdoor illuminance during daytime [26]. Otherwise the object is in a sunny position. If the virtual object is considered as in shadow then the $E_{D H}$ is set to 0 and $E_{K H}$ is set to $E$, otherwise the direct and diffuse illuminance values remain unchanged. In the current implementation it is considered that the virtual objects and the mobile device are under the same illumination conditions.

The process is dynamic and if an object is in the sun and a shadow is being casted and a cloud appears the system will detect the significative change in the values and would register the object has being in the shade and thus casting no shadow. The time for the system to react would be less than 1 second. This algorithm ensures that no shadows are created by the rendering process when the object is in shadow, thus allowing to handle the double shadow issue, i.e., when a shadow cast by a virtual object is rendered on the top of an already existing real shadow. Figure 2 shows the detection of dynamic shadows algorithm fluxogram.

\section{Rendering}

After the actual illuminance percentage of the sky and Sun have been estimated, the augmented objects can be rendered. An overview of our rendering pipeline is shown in Figure 3. The direct beam illuminance from the Sun is modelled using Lambert's cosine law as directional light. The sky diffuse illuminance is a hemisphere that surrounds us with the same radiance in all directions (sky dome). In this case, the intensity is constant and is thus not affected by anything in the scene or the user's position.

\subsection{Intensity adjustment}

The illumination model consists of a directional light source and ambient lighting. The estimated illuminance from the

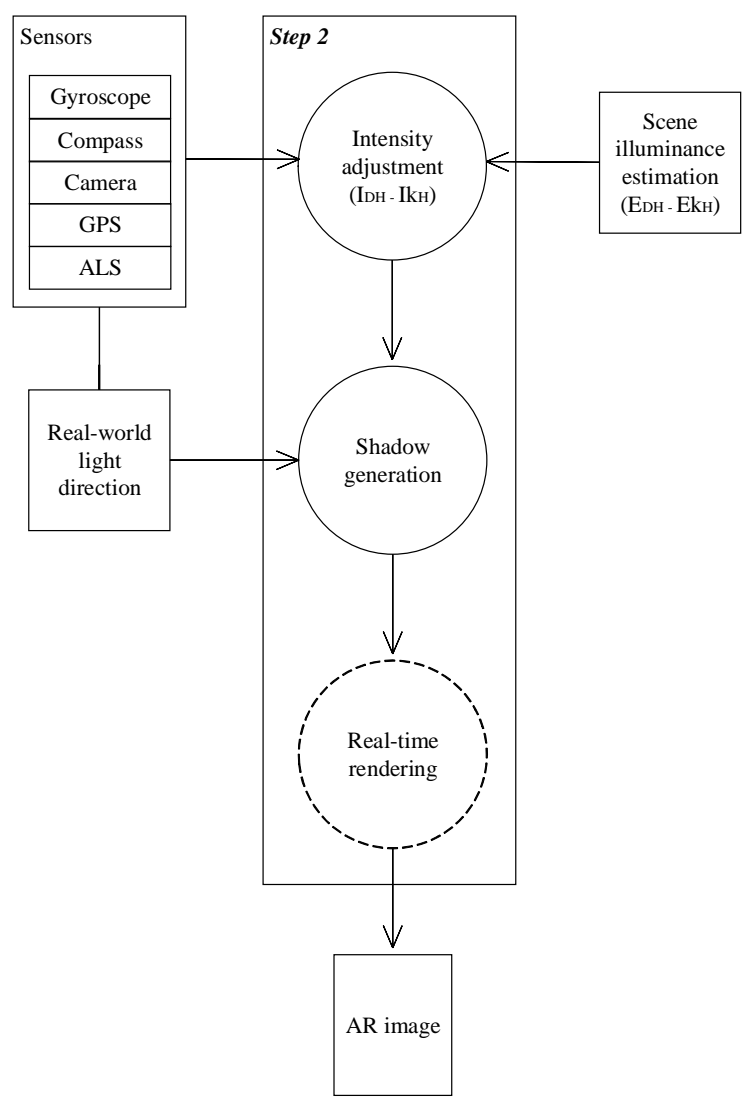

Fig. 3: Rendering pipeline. The scene illumination estimation from Step 1 is used to derive intensities for rendering. Shadows are then generated to appear consistently in the final AR image.

sky and Sun are normalized to intensities using the following equations:

$$
\begin{gathered}
I_{D H}=\frac{E_{D H}}{E_{T H}}, E_{D H} \leq E_{T H} \\
I_{K H}=\frac{E_{K H}}{E_{T H}}, E_{K H} \leq E_{T H}
\end{gathered}
$$

where $I_{D H}$ is an intensity value between $[0,1]$ used to adjust the virtual directional light and $I_{K H}$ is an intensity value between $[0,1]$ used to adjust the ambient light. $E_{D H}$ $E_{K H}$ is the illuminance value in lux due to the Sun or sky computed using our method. $E_{T H}$ is the typical maximum illuminance value (threshold) that can occur during each type of sky condition [25] (Table 2). Because bright sunlight is approximately $110,000 \mathrm{~lx}$, we optimize the reference value to the range of the light sensor $(32,768 \mathrm{~lx})$ as no significant difference can be perceived when exceeding this value. For example, if the absolute scene illumination $E$ reading from the ALS is $10,000 \mathrm{~lx}$ on a clear day (thus $E_{D H}=8,500 \mathrm{~lx}$ and $E_{K H}=1,500 \mathrm{~lx}$ from Equations (16) (17)), the directional light intensity and ambient light would be $0.26(8,500 / 32,768)$ and $0.05(1,500 / 32,768)$, respectively.

\subsection{Shadow generation}

Shadows are critical to maintain the illusion that virtual objects are seamlessly integrated into the real environment. 

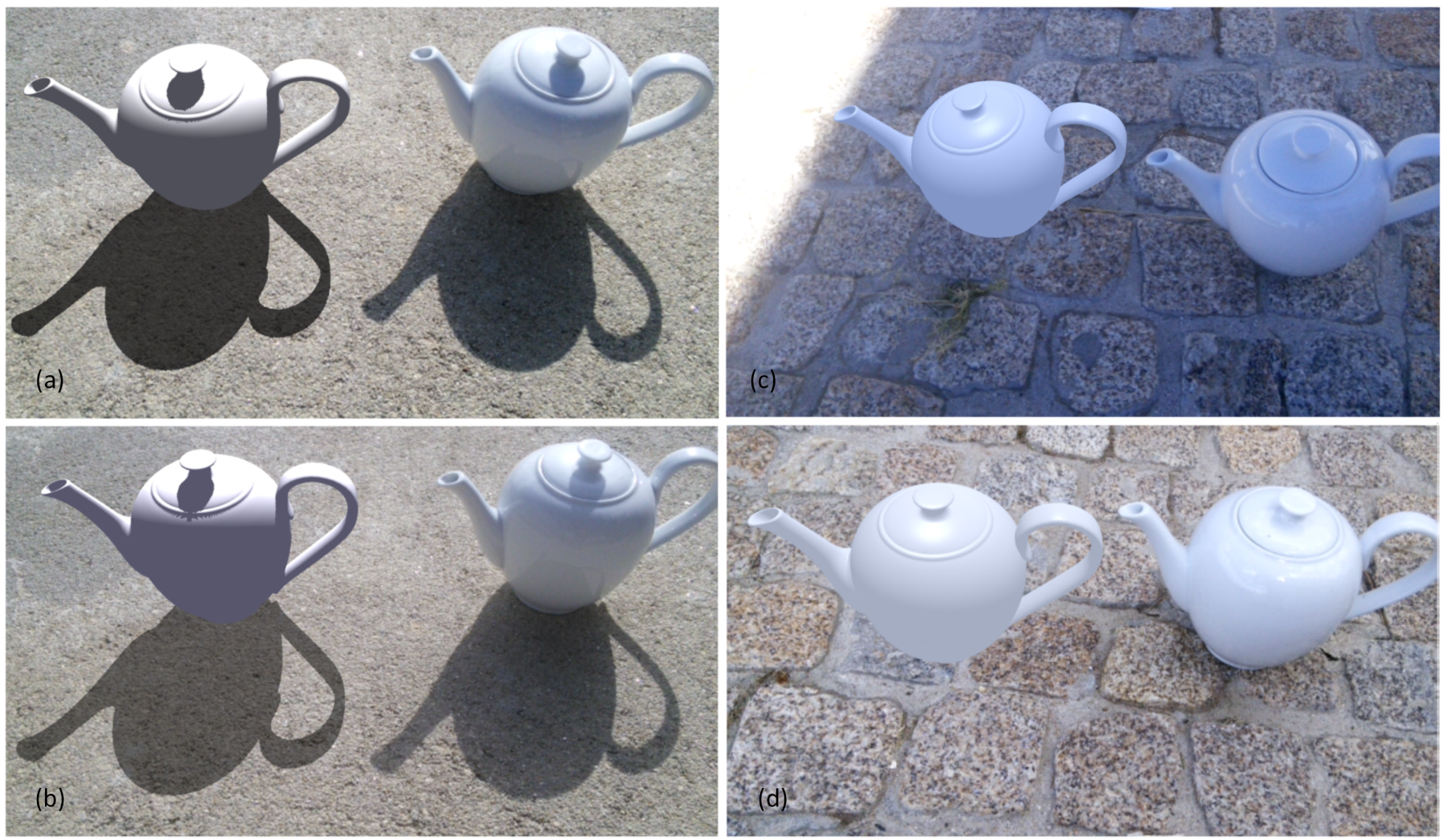

Fig. 4: Real-world results built with our method. Each image shows a virtual teapot (on the left) and a real teapot (on the right). Scene (a) shows direct illumination and clear sky. Scene (b) shows a different time of day during partly cloudy sky conditions, and scene (c) shows the objects in the shade. Scene (d) shows the objects rendered during overcast conditions.

TABLE 1: Threshold daylight intensities for each sky condition [25].

\begin{tabular}{lc}
\hline Sky condition & lux \\
\hline Overcast & 2000 \\
Partly cloudy & 25000 \\
Clear sky & 32768 \\
\hline
\end{tabular}

The shading model computes a virtual light of infinite size and distance that illuminates all objects in the scene equally from the calculated direction of the Sun. The camera position is estimated with the gyroscope data and the accelerometer for gravitational direction. The roll-angle data are used in the $\mathrm{x}$-axis orientation matrix, and the compass-heading angle data are used for the y-axis rotation. A fragment shader draws the object in a shadow or lighted depending on the result. The intensity of the shadows is calculated using the appropriate intensity factor estimated in equations (17)(18). Once the camera position is estimated, the shadows can be generated. The approach proposed in the previous step is used to prevent virtual objects from casting shadows where real shadows are already present.

\section{Evaluation}

This section presents preliminary evaluation of our method under natural lighting conditions in the real world. The visual results mainly focus on plausible lighting and shadows rather than on physically based results. The geometry is rendered using our method, which calculates the Sun and sky contributions. In outdoor scenes, the majority of the surfaces are diffuse (e.g., pavement, asphalt), and the light reflection is ideal and proportional to the incident irradiance from all directions.

We used traditional markers and Unity 3D for rendering to build the AR scene. All of the experiments were implemented on a Nexus 7 (2013) equipped with the required sensors and connected to a data network.

\subsection{Real-world results}

First, we investigated the effectiveness of the method under typical outdoor weather conditions. Figure 4 shows the effects of applying our approach for estimating the scene illuminance parameters in an AR scene. The system dynamically estimated the ambient lighting conditions using geospatial location, date and time and current atmospheric data. All of the scenes were exposed to unprepared natural environments with moving clouds. Scene (a) shows real and virtual teapots rendered in direct illumination. Scene (b) shows the same objects rendered at a different time of day during partly cloudy conditions. In scene (c), the objects are in the shade. Scene (d) shows the objects rendered under overcast sky conditions. As the teapot is a complex object composed of porcelain with shiny and translucent properties, we also used a parallelepiped with diffuse material for comparison. Figure 6 provides the results with a diffuse object in the real world. 

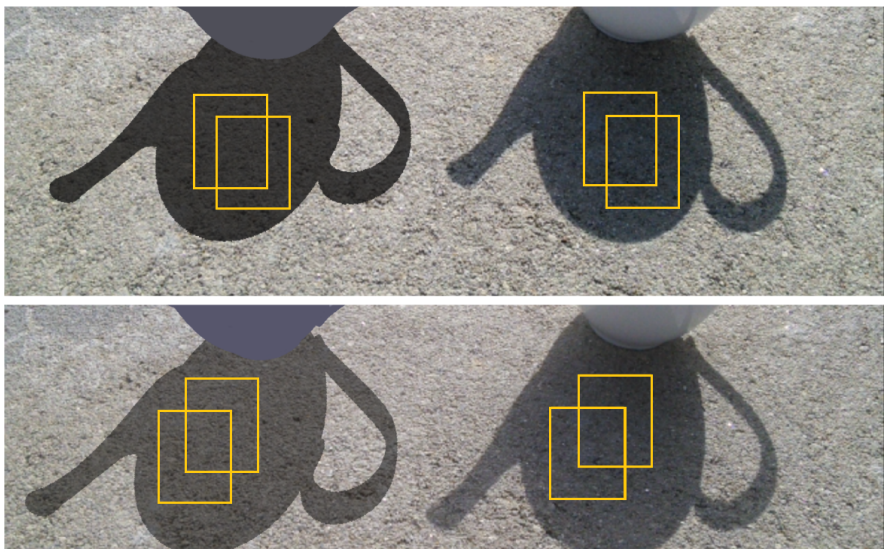

Fig. 5: Randomly selected regions.

TABLE 2: Illuminance measurements of the ALS and error percentage compared to reference.

\begin{tabular}{lccccc}
\hline & Reference & & & & \\
& $0^{\circ}$ & $-25^{\circ}$ & $-45^{\circ}$ & $-75^{\circ}$ & $-90^{\circ}$ \\
\hline Clear (lx) & 32768 & 29500 & 28100 & 7505 & 6100 \\
Error & - & $10 \%$ & $14 \%$ & $77 \%$ & $81 \%$ \\
\hline Partly (lx) & 3200 & 3100 & 2700 & 880 & 700 \\
Error & - & $2.5 \%$ & $15 \%$ & $73 \%$ & $78 \%$ \\
\hline Cloudy (lx) & 700 & 700 & 700 & 700 & 700 \\
Error & - & - & - & - & - \\
\hline
\end{tabular}

\subsection{Influence of orientation}

The absolute scene illuminance was estimated through the ALS of the device, which depended on the orientation. To capture the absolute scene illuminance as precisely as possible, we set the actual field of view to an angle of $90^{\circ}$ from the horizontal. The reference was the position of the device where the ALS was not obstructed. As the sensor was facing the same direction as the display (i.e., facing the user), the ALS reading increased when the world-facing camera was pointed toward the floor. Table 3 shows the captured illumination information. The errors were small under the defined angle intervals (i.e., $0^{\circ},-25^{\circ}$ and $-45^{\circ}$ ) for the approach, thus illustrating that the proposed approach allowed for reliable measurements of the light intensity. The captured illuminance did not depend on the orientation during an overcast day because only the diffused illumination from the sky illuminated the scene.

\subsection{Quantitative evaluation}

We also performed a quantitative evaluation by comparing the estimated intensities of the shadows against ground truth from the real-world images in Figure 4. We calculated the average difference between two randomly selected regions of the real and virtual shadows (Figure 5). The errors were small-approximately $15-25 \%$ in each scene.

\subsection{Case study: coherence of the shadows with the user's perception}

To better understand a user's perception of the shadows generated by the proposed method in terms of their shape and color, we performed a study comparing a naive method and the proposed method. Both methods used the same geometrical model. In the naive method, the context was not considered, and thus, the illuminance parameters were not estimated and were instead set to average values in a day with the current sky conditions. The Sun's position was set to the $2^{\text {nd }}$ moment of the day. In our method, all of these parameters were automatically calculated and considered.

\subsubsection{Materials}

The test scenario consisted of a real parallelepiped shown on the right side and the same virtual object appearing on the left, which was rendered using the proposed method or the naive method. Four time frames were considered, corresponding to three different hours of the day $(12 \mathrm{pm}$, $1: 30 \mathrm{pm}$ and $3 \mathrm{pm}$ ) with the object in sunlight and one moment with the object in the shadow (Figure 6). Thus, 8 experimental conditions were considered in total (i.e., 4 time frames $x 2$ rendering methods). It is difficult to guarantee that each participant would run the experiment under the same experimental conditions for each time frame. Thus, a photograph of the AR application running on screen for each experimental condition was taken instead of using a live AR application. All photographs were taken on a clear day in December.

In the naive method, the context information was not considered, so the shadows of the rendered virtual object do not change in the first three images. Our proposed method considered the context and thus generated different shadows when rendering the virtual object.

An online questionnaire displaying the eight images was prepared to collect the data. There were three questions for each image: Choose one of the following. In the shown image, the

- left object is VIRTUAL, and the right object is REAL.

- left object is REAL, and the right object is VIRTUAL

- both objects are REAL.

- both objects are VIRTUAL.

Regarding the shadows in the image, quantify the degree of coherence between the shapes of the objects. (The answer is given on a 10-point Likert scale, where 1 corresponded to no coherence and 10 corresponded to entirely coherent.)

Regarding the shadows in the image, quantify the degree of coherence between the colors of the shadows. (The answer is given on a 10-point Likert scale, where 1 corresponded to no coherence and 10 corresponded to entirely coherent.)

\subsubsection{Participants}

The study included 20 participants (10 males and 10 females) between the ages of 22 and 49 years old ( $M=28.65$, $\mathrm{SD}=7.34)$. All participants reported normal or corrected-tonormal vision.

\subsubsection{Procedure}

Each participant was invited to participate in the study by email, where we explained the purpose of the study and provided all necessary instructions. We also explained that by answering the questionnaire, they were also giving their consent to participate in the study. After answering all of the questions, the participants submitted the forms and the data were automatically stored. 


\subsubsection{Results and discussion}

The majority of the participants (at least $80 \%$ ) correctly identified the object on the left as virtual and the object on the right as real. Only 4 participants had difficulty determining whether the objects were virtual or real. Nevertheless, these results had no impact on the shadow comparison. For each image, the participants classified the degree of visual coherence between the objects' shadows in terms of shape and color.

In terms of shape, the median scores of the proposed method for the $1^{\text {st }}, 2^{\text {nd }}$ and $3^{\text {rd }}$ moments of the day were $8.00,8.00$ and 7.00 , respectively. For the naive rendering method, the median scores at the considered moments of the day were 2.50, 6.00 and 1.00, respectively. A Wilcoxon signed-rank test showed that the differences between the scores for each rendering method at $12 \mathrm{pm}$ $(\mathrm{Z}=-3.833 ; \mathrm{p}<0.001), 1: 30 \mathrm{pm}(\mathrm{Z}=-2.995 ; \mathrm{p}=0.003)$ and $3 \mathrm{pm}$ $(Z=-3.954 ; p<0.001)$ were significant. The proposed method consistently had better scores than the naive method.

In terms of color, the median scores of the proposed method for the $1^{s t}, 2^{\text {nd }}$ and $3^{\text {rd }}$ moments of day were $7.00,7.00$ and 6.50 , respectively. For the naive rendering method, the median scores at the considered moments of the day were 5.00, 8.00 and 5.00, respectively. For these scores, we also performed a Wilcoxon signed-rank test to assess whether the differences were significant. The proposed method had better ratings than the naive method in the $1^{\text {st }}$ moment of the day $(\mathrm{Z}=-2.440 ; \mathrm{p}=0.015)$ and in the $3^{\text {rd }}$ moment of the day $(Z=-0.246 ; p=0.022)$. However, the naive method obtained fair rates in this situation $(>5)$. In the $2^{\text {nd }}$ moment of the day, both methods had similarly good ratings $(\mathrm{Z}=-2.285 ; \mathrm{p}=0.806>0.05)$.

In the $4^{\text {th }}$ time frame the proposed method, as expected, did not generate the object's shadow, while the naive method could not detect that the object is in shadow and wrongly generated the corresponding shadow and thus did not correct the double shadow problem (Figure 6, last row). We did not ask the participants to compare the images of the $4^{\text {th }}$ time frame because the shadows were only visible in one of the images, which could have confused the participants as they were asked to compare visible shadows.

In this experiment, the proposed method consistently obtained good ratings in terms of the shape and color of the projected shadow and performed consistently better than the naive method. This result illustrates that generating consistent shadows is important to improving the visual coherence of the projected shadows.

\section{LIMITATIONS}

Although the results showed that our method has better results than a naive method. The proposed method still has limitations. Next we describe the main limitations of the system and discuss possible solutions to overcome them.

Detection/Shading: Currently the method is not capable of correctly represent an object that is only halfway in the shade. It will represent the object that has been completely in the shadow or completely in the light and the object must be in the same conditions the mobile device is. This limitation can only be solved using other approaches namely by making use of computer vision techniques to analyze the

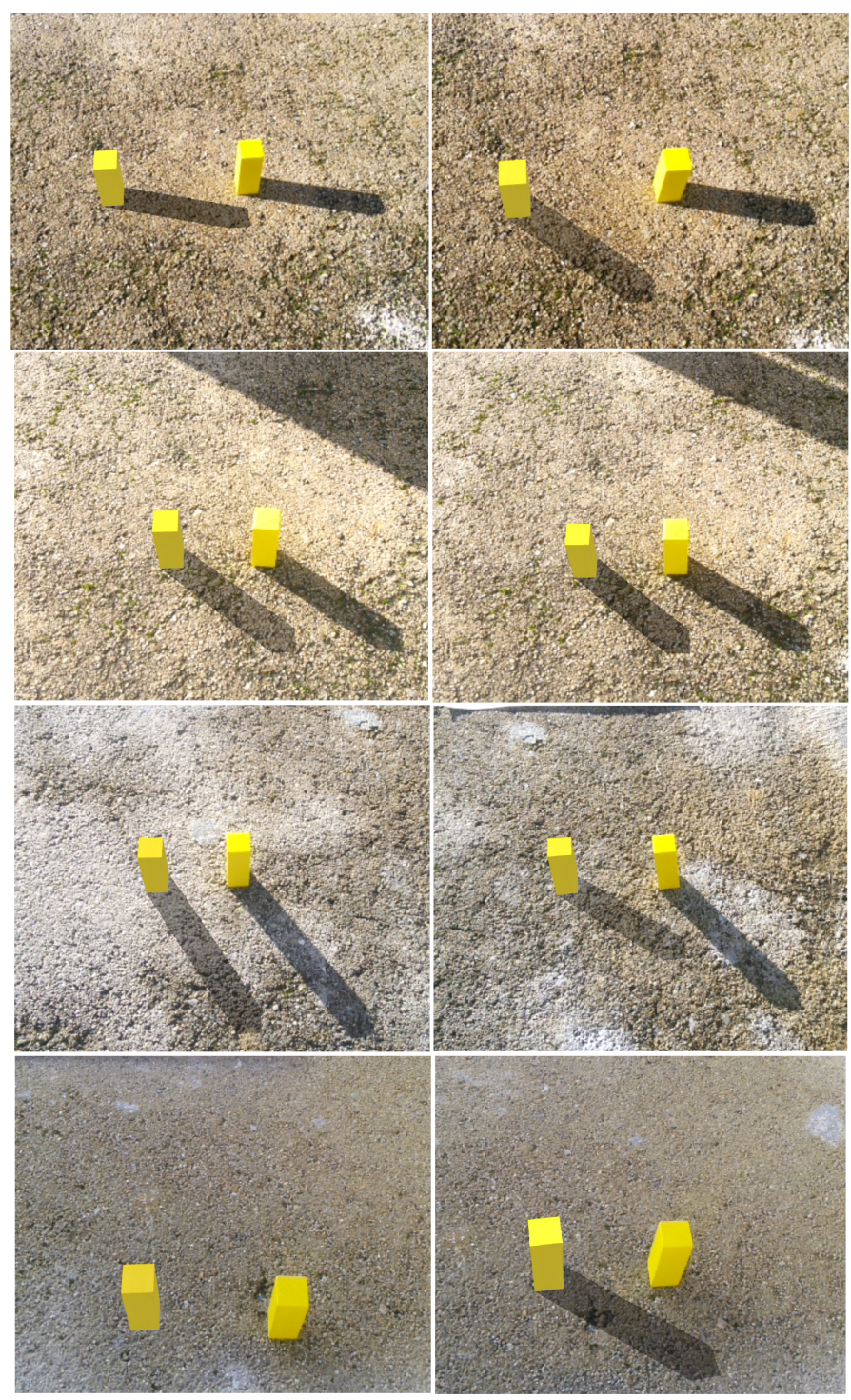

Fig. 6: Real-world results with a diffuse object. The images show a real parallelepiped on the right side. The same virtual object appears on the left rendered at three different hours of the day (12pm, 1:30pm and $3 \mathrm{pm})$ using the proposed method (left column) and the naive method (right column).

scene and provide the information required for the method to work.

Given that, in this first phase, our main objective was to experiment if with the data available just from the context, it was possible to achieve a coherent representation of a virtual shadow. In this first approach we only considered one light source and one virtual object at a time. In future work we will consider having other objects in the scene that cast multiple shadows and coherently represent them.

Another limitation is the parameters estimates as the device moves around. As we can see in table 2 the error associated with the sensor is high if the angle is superior to $-75^{\circ}$ when someone is moving around it is not easy to hold the mobile device in the correct angle and thus the estimates can vary significantly. This could easily be improved by having more than one sensor in the mobile device. 
Color correction: The color correction of the objects can be nonlinear because physical rendering of a radiometric calibration would be required with the recovered scene irradiance and camera settings, which greatly increases the complexity of the system. Although the method is fully automated, we plan to extend our system with controls that allow the user to calibrate the color correction.

Indoor: Currently the method is only suitable for outdoor scenes. In the future, we will also extend the system for indoor scenarios by capturing the front-side camera image of the device in conjunction with the orientation sensor to estimate the light intensity and direction.

\section{CONCLUSION}

In this paper, we presented a novel method to estimate environmental lighting using embedded sensors available on current mobile platforms. Our method does not rely on a priori or recovered knowledge of the scene, or on the use of intrusive objects, as do existing methods. This makes our method suitable to be used on unprepared outdoor environments. Moreover, it does not use any image processing technique to estimate the illumination parameters, making it a simple and efficient method that could be easily implemented on a mobile device and used within real-time AR applications.

Although the results, both objective and subjective, indicate that our method, based on context-aware live sensors data, generates visually coherent AR images, the algorithm still needs to be tested in detail in real applications which include more complex environments. In addition, a number of severe limitations still exist. Most importantly, the system is not yet able to determine whether the mobile device operates under the same lighting conditions as the virtual object. Furthermore, it is not yet possible to estimate the absolute illuminance. In this paper, the estimation of the absolute illuminance using mobile sensors is currently prone to errors due to how the mobile device is held. These problems will be addressed in future work.

\section{ACKNOWLEDGMENTS}

This work was supported in part by the Fundação para a Ciência e Tecnologia - FCT, under the grant number SFRH/BD/73129/2010, and the European Union (COMPETE, QREN and FEDER), under the project REC I/EEISII/0360/2012 "MASSIVE - Multimodal Acknowledgeable MultiSenSorial Immersive Virtual Environments". This work is also supported by the project "TEC4Growth Pervasive Intelligence, Enhancers and Proofs of Concept with Industrial Impact/NORTE-01-0145-FEDER-000020" is financed by the North Portugal Regional Operational Programme (NORTE 2020), under the PORTUGAL 2020 Partnership Agreement, and through the European Regional Development Fund (ERDF).

\section{REFERENCES}

[1] R. Azuma, Y. Baillot, R. Behringer, S. Feiner, S. Julier, and B. MacIntyre, "Recent advances in augmented reality," Computer Graphics and Applications, IEEE, vol. 21, no. 6, pp. 34-47, nov/dec 2001.
[2] R. A. Newcombe, S. Izadi, O. Hilliges, D. Molyneaux, D. Kim, A. J. Davison, P. Kohli, J. Shotton, S. Hodges, and A. Fitzgibbon, "Kinectfusion: Real-time dense surface mapping and tracking," in IEEE ISMAR. IEEE, October 2011.

[3] Y. Yu, P. Debevec, J. Malik, and T. Hawkins, "Inverse global illumination: recovering reflectance models of real scenes from photographs," in Proceedings of the 26th annual conference on Computer graphics and interactive techniques, ser. SIGGRAPH '99. New York, NY, USA: ACM Press/AddisonWesley Publishing Co., 1999, pp. 215-224. [Online]. Available: http://dx.doi.org/10.1145/311535.311559

[4] P. Debevec, "Rendering with natural light," in ACM SIGGRAPH 98 Electronic art and animation catalog, ser. SIGGRAPH '98. New York, NY, USA: ACM, 1998, pp. 166-. [Online]. Available: http://doi.acm.org/10.1145/281388.281983

[5] T. Grosch, T. Eble, and S. Mueller, "Consistent interactive augmentation of live camera images with correct near-field illumination," in Proceedings of the 2007 ACM symposium on Virtual reality software and technology, ser. VRST '07. New York, NY, USA: ACM, 2007, pp. 125-132. [Online]. Available: http://doi.acm.org/10.1145/1315184.1315207

[6] M. Aittala, "Inverse lighting and photorealistic rendering for augmented reality," The Visual Computer, vol. 26, no. 6-8, pp. 669678, 2010. [Online]. Available: http://dx.doi.org/10.1007/s00371010-0501-7

[7] D. A. Calian, K. Mitchell, D. Nowrouzezahrai, and J. Kautz, "The shading probe: fast appearance acquisition for mobile ar," in SIGGRAPH Asia 2013 Technical Briefs. ACM, 2013, p. 20.

[8] M. Knecht, C. Traxler, O. Mattausch, W. Purgathofer, and M. Wimmer, "Differential instant radiosity for mixed reality," in Mixed and Augmented Reality (ISMAR), 2010 9th IEEE International Symposium on, Oct 2010, pp. 99-107.

[9] P. Kan and H. Kaufmann, "High-quality reflections, refractions, and caustics in augmented reality and their contribution to visual coherence," in Proceedings of the 2012 IEEE International Symposium on Mixed and Augmented Reality (ISMAR), ser. ISMAR '12. Washington, DC, USA: IEEE Computer Society, 2012, pp. 99-108. [Online]. Available: http:/ /dx.doi.org/10.1109/ISMAR.2012.6402546

[10] K. Karsch, V. Hedau, D. Forsyth, and D. Hoiem, "Rendering synthetic objects into legacy photographs," in ACM Transactions on Graphics (TOG), vol. 30, no. 6. ACM, 2011, p. 157

[11] K. Karsch, K. Sunkavalli, S. Hadap, N. Carr, H. Jin, R. Fonte, M. Sittig, and D. Forsyth, "Automatic scene inference for 3d object compositing," ACM Transactions on Graphics (TOG), vol. 33, no. 3, p. 32, 2014 .

[12] J. Jachnik, R. A. Newcombe, and A. J. Davison, "Real-time surface light-field capture for augmentation of planar specular surfaces," in Proceedings of the 2012 IEEE International Symposium on Mixed and Augmented Reality (ISMAR), ser. ISMAR '12. Washington, DC, USA: IEEE Computer Society, 2012, pp. 91-97. [Online]. Available: http://dx.doi.org/10.1109/ISMAR.2012.6402544

[13] J.-F. Lalonde, A. Efros, and S. Narasimhan, "Estimating natural illumination from a single outdoor image," in Computer Vision, 2009 IEEE 12th International Conference on, 29 2009-oct. 2 2009, pp. $183-190$.

[14] C. Madsen and B. Lal, Outdoor Illumination Estimation in Image Sequences for Augmented Reality. INSTICC Press, 2011, pp. 129 $-139$.

[15] Y. Wang and D. Samaras, "Estimation of multiple directional light sources for synthesis of mixed reality images," in Computer Graphics and Applications, 2002. Proceedings. 10th Pacific Conference on, 2002, pp. 38-47.

[16] I. Sato, Y. Sato, and K. Ikeuchi, "Illumination from shadows," Pattern Analysis and Machine Intelligence, IEEE Transactions on, vol. 25, no. 3, pp. 290-300, March 2003.

[17] Y. Liu, X. Qin, G. Xing, and Q. Peng, "A new approach to outdoor illumination estimation based on statistical analysis for augmented reality," Computer Animation and Virtual Worlds, vol. 21, no. 3-4, pp. 321-330, 2010. [Online]. Available: http://dx.doi.org/10.1002/cav.357

[18] Y. Liu and X. Granier, "Online tracking of outdoor lighting variations for augmented reality with moving cameras," Visualization and Computer Graphics, IEEE Transactions on, vol. 18, no. 4, pp. 573 -580 , april 2012.

[19] S. B. Knorr and D. Kurz, "Real-time illumination estimation from faces for coherent rendering," in Mixed and Augmented Reality 
(ISMAR), 2014 IEEE International Symposium on. IEEE, 2014, pp. $113-122$

[20] L. Gruber, T. Richter-Trummer, and D. Schmalstieg, "Real-time photometric registration from arbitrary geometry," in Mixed and Augmented Reality (ISMAR), 2012 IEEE International Symposium on, Nov 2012, pp. 119-128.

[21] L. Gruber, T. Langlotz, P. Sen, T. Hoherer, and D. Schmalstieg, "Efficient and robust radiance transfer for probeless photorealistic augmented reality," in Virtual Reality (VR), 2014 iEEE, March 2014, pp. $15-20$.

[22] M. B. Muriel, D. A. Padilla, T. L. Moratalla, and M. Lara-Coira, "Computing the solar vector," Solar Energy, vol. 70, no. 5, pp. 431 $-441,2001$.

[23] J. J. Michalsky, "The astronomical almanac's algorithm for approximate solar position (1950-2050)," Solar energy, vol. 40, no. 3, pp. 227-235, 1988.

[24] IESNA, Recommended Practice for the Calculation of Daylight Availability. The Society, 1984. [Online]. Available: http://books.google.pt/books?id=8WdDHQAACAAJ

[25] R. Bird, R. Hulstrom, S. E. R. Institute, and U. S. D of Energy, A Simplified Clear Sky Model for Direct and Diffuse Insolation on Horizontal Surfaces, ser. SERI/TR. Solar Energy Research Institute, 1981. [Online]. Available: http:/ / books.google.pt/books?id=E92rGAAACAAJ

[26] R. R. Hainich and O. Bimber, Displays: Fundamentals and Applications, 1st ed. Natick, MA, USA: A. K. Peters, Ltd., 2011.

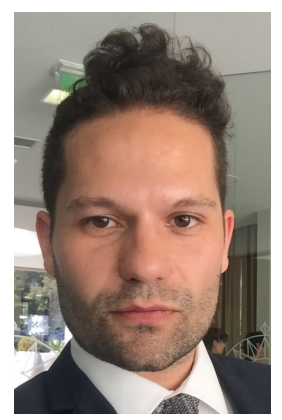

João Barreira received the $\mathrm{PhD}$ degree in Computer Science from the Universidade de Trásos-Montes e Alto Douro, Vila Real, Portugal, in 2016. His research interests include Augmented Reality, Context-awareness, and Multisensory in mixed environments.

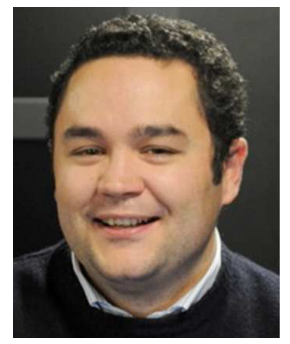

Maximino Bessa is an Assistant Professor in Computer Science at the Engineering Department of University of Trás-os-Montes e Alto Douro, Portugal. He obtained his PhD degree in Computer Science from the University of Trás-os-Montes e Alto Douro whose dissertation theme was Selective Rendering for High-Fidelity Graphics for 3D Maps on Mobile Devices. His area of interest and research are mainly Computer Graphics, HDR, Multisensory Virtual Environments and he has participated in several related research projects. He has several publications including books, refereed publications, and communications at international conferences.

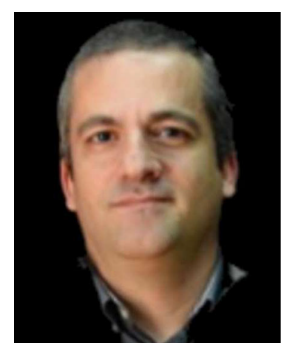

Luís Barbosa is a computer science teacher at the Engineering department of the University of Trás-os-Montes e Alto Douro, Portugal. He obtained his $\mathrm{PhD}$ degree in Computer Science from the University of Trás-os-Montes e Alto Douro whose dissertation theme was Teaching Support Information Systems The Case Study of the portal SIDE from UTAD. His area of interest and research are mainly Information Systems, Multisensory Virtual Environments and Interaction and has participated in several related re-

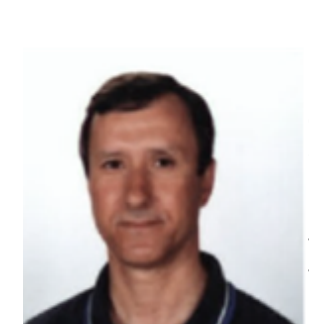

Luís Magalhães has a BSc and MSc in Informatics from the University of Minho and a PhD in Computer Science from the University of Trásos-Montes e Alto Douro. Currently, he is an Assistant Professor with Habilitation at University of Minho. He is also a Senior Researcher at ALGORITMI Centre. He is author or co-author of more than 70 scientific publications. His research interests include the use of Computer Vision, Augmented Reality, Computer Graphics, Procedural Modeling, High Dynamic Range Imaging. He was a research member in projects related to Expeditious Modeling, Immersive 3D Environments, Virtual Heritage and Mixed Reality Systems.

search projects. 